\title{
Giant Left Atrial Appendage Aneurysm: Surgical Treatment to Prevent Potential Complications
}

\author{
Peng Teng, MD ${ }^{1}$ Yiming Ni, MD, ${ }^{1}$ Qianhui Sun, ${ }^{2}$ Haige Zhao, $M D^{1}$ \\ Departments of ${ }^{1}$ Cardiothoracic Surgery and ${ }^{2}$ Surgical Intensive Care Unit, First Affiliated Hospital of Zhejiang University, \\ Hangzhou, China
}

\section{ABSTRACT}

Left atrial appendage aneurysm (LAAA) is a rare anomaly, which predisposes to hazardous cardiovascular events. We present a rare case of a 26-year-old female with a giant left atrial appendage aneurysm. The left atrial appendage was incidentally detected during her pregnancy. Six months later after her parturition, the patient was referred to our department and successfully received surgical treatment prophylactically to prevent potential complications. To the best of our knowledge, only about 100 cases of LAAA have been reported.

\section{INTRODUCTION}

Left atrial appendage aneurysm (LAAA) is a rare anomaly, commonly associated with potentially fatal complications like atrial arrhythmia, thromboembolic event, compression of surrounding structures and cardiac dysfunction. Surgical treatment is necessary and generally offers promising outcomes. Because of the risk of life-threatening complications, it is important to recognize this disease in an early period and perform timely intervention.

\section{CASE REPORT}

A 26-year-old female was referred to our department for incidental detection of a pericardial cyst by echocardiography during her pregnancy. The patient was asymptomatic and remained in sinus rhythm. Physical examination and laboratory tests were unremarkable.

On admission, chest roentgenogram showed cardiomegaly with a prominent upper left heart border. Transthoracic echocardiography (TTE) revealed a giant $(8.7 \times 5.0 \mathrm{~cm})$ cystic structure arising from the left atrium (LA). It compressed the anterolateral left ventricular wall during the entire cardiac cycle (Figure, A). Further transesophageal echocardiography (TEE) confirmed the structure communicated with the LA by a $2.8 \times 2.0 \mathrm{~cm}$ entrance. Enhanced computed tomography

Received fuly 27, 2018; accepted September 25, 2018.

This work was supported by Zhejiang Medical and Health Science and Technology Project (2018249646).

Correspondence: Haige Zhao, \#79 Qingchun Road, Hangzhou, Zhejiang, China, 310000; +860571 87236841; fax: +860571 87236843 (e-mail: haigezhao@zju.edu.cn).
(CT) showed the structure caused slight compression effect on the left ventricle (LV) (Figure, B and C).

A preoperative diagnosis of the LAAA was made based on its cystic structure and communication with the LA. Given the potential risk of adverse events, we advised surgical treatment.

On-pump aneurysmectomy of the LAAA with beating heart was scheduled. A giant LAAA (about $8 \times 7 \mathrm{~cm}$ ) was noted via median sternotomy after pericardiotomy. Conventional cardiopulmonary bypass (CPB) was established by aortic and right atrial cannulation. After the LAAA was completed exposed, the pump flow was reduced and hypovolemic condition was achieved in a short period, which can reduce the tension of the left atrial appendage (LAA) wall and reduce the risk of dehiscence and delayed hemorrhage. The LAAA was successfully resected by a straight stapler along with the base on a beating heart. The stump of the LAAA was
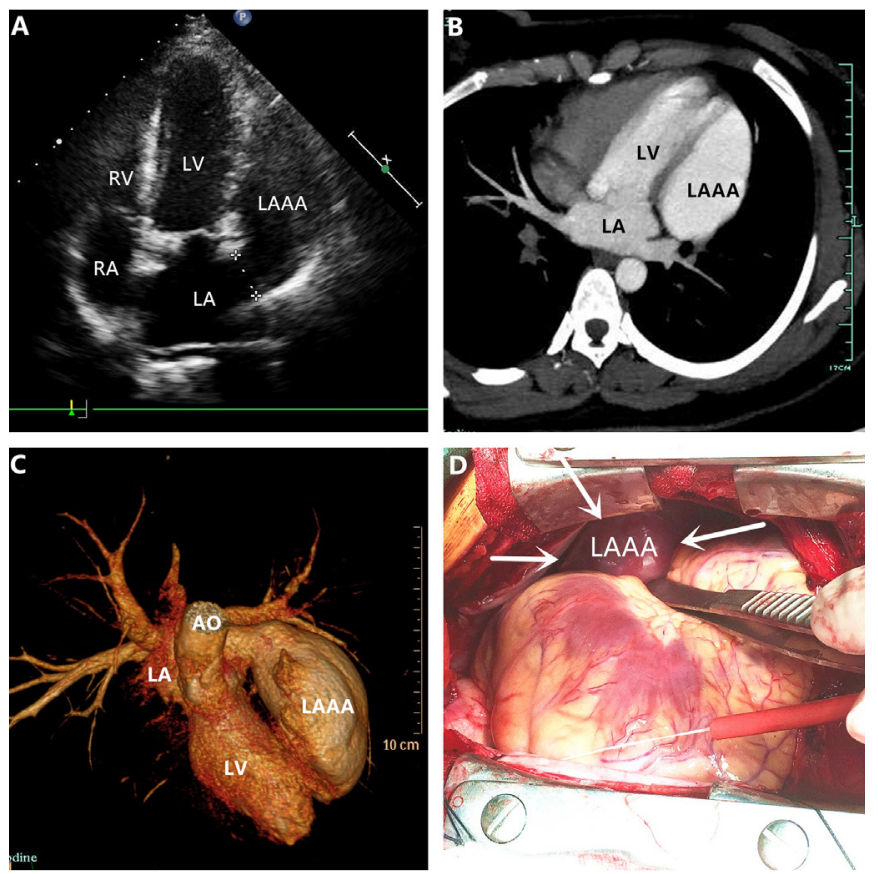

$A$, TTE showed a giant LAAA, arising from the LA, compressing the LV. B, Enhanced CT confirmed the diagnosis of LAAA and the LV compression caused by LAAA. C, Three-dimensional reconstruction of enhanced $C T$ confirmed the diagnosis of LAAA and its relationship with surrounding structures. D, Intraoperative view on the giant LAAA. 
reinforced by continuous suture. The resected LAAA had a thin wall and was hypocontractile with no thrombus inside. The pathological examination confirmed the diagnosis of LAAA. The patient had an uneventful recovery without any complications and has been followed up for 2 years without any sign of recurrence.

\section{DISCUSSION}

LAAA is a rare entity which is generally considered to be either congenital, caused by dysplasia of musculi pectinati or partial absence of the pericardium, or acquired as a result of mitral valve disease or syphilitic myocarditis [Chowdhury 2009]. To the best of our knowledge, only about 100 cases have been reported in the literature since the first description by Parmley in 1962 during a surgical procedure [Aryal 2014]. According to our literature review, two features have been observed: (1) slight female predominance, and (2) most cases are diagnosed between the second and fourth decades of life [Hosseini 2016].

Palpitation and/or dyspnea is the most common symptom, followed by systemic thromboembolic events and chest pain. However, it is hard to discern if these symptoms have a cause-effect relationship with LAAA or are simply a manifestation of some other cardiac anomalies. A recent study demonstrated that aneurysm size did not predict thrombus formation or thromboembolic events. Only $10-20 \%$ of patients remain asymptomatic and are diagnosed incidentally.

Based on the study by Aryal et al, the proposed definition of LAAA was an LAA that has dimensions at the diameter of the orifice, with width and the length of the LAA larger than 2.7, 4.8, and $6.8 \mathrm{~cm}$ respectively [Aryal 2014].

Comprehensive evaluation by echocardiography, enhanced CT, and MRI is necessary and helpful to confirm the diagnosis and clarify relationships with surrounding structures. The common findings on chest roentgenogram are cardiomegaly or a local prominent left heart border. TTE is the most important screening tool but with limited value in diagnosis. TEE is necessary and superior to TTE for the diagnosis of LAAA. MRI and enhanced CT can be helpful to confirm the diagnosis and clarify relationships with surrounding tissue like left pulmonary veins and circumflex artery [Hoffmann 2000].
The natural history of LAAA is unpredictable and considered to be associated with high risk of arrhythmia and thromboembolic events. Patients undergoing surgical resection usually have a favorable prognosis, as none of the patients report recurrent aneurysm or symptoms. Once LAAA is diagnosed, surgical resection is always recommended, even in asymptomatic patients. Conventional surgical procedure is aneurysmectomy or LAA resection with CPB through sternotomy. In our case, we chose on-pump technique mainly due to inexperience and fear of stump dehiscence. We believe CPB without cardioplegia can balance the surgical risk and myocardial injury. Other surgical methods include endoscopic resection, percutaneous LA occlude device, and tourniquet snare techniques. In some cases, an additional Cox-Maze procedure or simply pulmonary vein isolation is reasonable when patients are in atrial fibrillation [Mathur 2005].

\section{CONCLUSION}

LAAA is a rare cardiac anomaly with potentially hazardous adverse events. This case report describes a rare giant LAAA incidentally diagnosed during pregnancy, which has seldomly been reported before. Comprehensive evaluation by echocardiography, enhanced CT, and MRI is necessary and helpful for diagnosis. Once the aneurysm is diagnosed, surgical treatment is highly recommended, even in asymptomatic patients because of its favorable long-term outcomes.

\section{REFERENCES}

Aryal MR, Hakim FA, Ghimire S, et al. 2014. Left atrial appendage aneurysm: a systematic review of 82 cases. Echocardiography 31:1312-8.

Chowdhury UK, Seth S, Govindappa R, Jagia P, Malhotra P. 2009. Congenital left atrial appendage aneurysm: a case report and brief review of literature. Heart Lung Circ 18:412-6.

Hoffmann U, Hamed N, Herold C, Globits S. 2000. Radiological signs of a left atrial aneurysm. Eur Radiol 10:1332-4.

Hosseini S, Hashemi A, Saedi S, et al. 2016. Left atrial appendage aneurysm. Ann Thorac Surg 102:e207-9.

Mathur A, Zehr KJ, Sinak LJ, Rea RF. 2005. Left atrial appendage aneurysm. Ann Thorac Surg 79:1392-3. 